\title{
ProduCCIÓN DE ETANOL POR SACCHAROMYCES CEREVISIAE A PARTIR DE GLICERINA, SUBPRODUCTO DE BIODIESEL
}

Jorge Augusto Hernández-Mora ${ }^{1}$, Juan Camilo Acevedo-Páez ${ }^{2}$

${ }^{1}$ Docente investigador. Correo electrónico: jo.hernandez@mail.udes.edu.co

2 Docente investigador

Grupo de Investigación Eureka UDES. Facultad de Administración e Ingenierías, Universidad de Santander, sede Cúcuta

Recibido: 10 de septiembre del 2013. Aprobado: 5 de noviembre del 2013.

Cómo citar este artículo: J. A. Hernández-Mora y J. C. Acevedo-Páez, "Producción de etanol por Saccharomyces cerevisiae a partir de glicerina, subproducto de biodiesel”. Ingeniería Solidaria, Vol. 9, No. 16, pp. 97-101, Dic., 2013.

Resumen. Como resultado del creciente mercado del biodiesel, se ha producido un aumento inevitable en la oferta de glicerina obtenida como subproducto del proceso. Con un abundante suministro barato de la glicerina sin refinar en el mercado, los investigadores buscan encontrar nuevas alternativas de uso. Por ello, en esta investigación se pretende analizar cómo la glicerina proveniente de la producción de biodiesel de aceite de palma puede ser la materia prima para la producción de químicos con un alto valor comercial por medios fermentativos y ser base para futuras biorrefinerías. A partir de la mezcla de glicerina, con diferentes sustratos por medio de un proceso de fermentación microbiana anaeróbica a partir de una cepa de Saccharomyces cerevisiae y una separación posterior, se pueden obtener diferentes productos de alto valor agregado como el etanol.

Palabras clave: etanol, fermentación microbiana, glicerina, subproducto de biodiesel.

\section{Producing Ethanol from Glycerin, a Biofuel By-Product, Using SACCharomyces Cerevisiae}

Abstract. The growing biofuel market has led to an inevitable increase in the availability of glycerin, a by-product of the production process. Due to this abundant supply of cheap unrefined glycerin, researchers are looking for new alternative uses. This research analyzes how the glycerin that comes from the production of palm oil biofuel could be used as the raw material for producing chemicals with high commercial value through fermenting agents, thus providing the basis for future bio-refineries. By mixing glycerin with different substrates via an anaerobic microbial fermentation process using a strain of Saccharomyces cerevisiae and prior separation, different products with high added value, such as ethanol, can be obtained.

Keywords: ethanol, microbial fermentation, glycerin, biofuel byproduct.

\section{Produção de ETANOL POR SACCHAROMYCES CEREVISIAE A PARTIR DE GLICERINA, SUBPRODUTO DE BIODIESEL}

Resumo. Como resultado do crescente mercado do biodiesel, produziuse um aumento inevitável na oferta de glicerina obtida como subproduto do processo. Com um abundante fornecimento barato da glicerina sem refinar no mercado, os pesquisadores procuram encontrar novas alternativas de uso. Por isso, nesta pesquisa, pretende-se analisar como a glicerina proveniente da produção de biodiesel de óleo de palma pode ser a matéria-prima para a produção de químicos com um alto valor comercial por meios fermentativos e ser base para futuras biorrefinarias. A partir da mistura de glicerina com diferentes substratos por meio de um processo de fermentação microbiana anaeróbica a partir de uma cepa Saccharomyces cerevisiae e uma separação posterior, podem-se obter diferentes produtos de alto valor agregado como o Etanol.

Palavras-chave: etanol, fermentação microbiana, glicerina, subproduto de biodiesel. 


\section{Introducción}

Durante años, la base del desarrollo de los países industrializados ha sido el uso masivo de combustibles fósiles y hoy sigue siendo un elemento principal de los procesos de cambio económico que caracterizan a los países más poblados del mundo. Esta gran dependencia respecto al uso de combustibles fósiles ha generado históricamente una gran preocupación por los impactos ambientales de la combustión masiva de combustibles fósiles y, sobre todo, de sus efectos en el cambio climático.

A nivel mundial, durante los últimos años las reservas de crudo han experimentado una disminución considerable, mientras que la producción ha variado en función de la demanda y de las políticas de producción de la Organización de Países Exportadores de Petróleo (OPEP), según la Secretaría de Energía de México [1]. De igual manera, el mercado de combustibles fósiles en Colombia ha tenido un comportamiento similar al del mercado mundial. Por una parte, el volumen de reservas de crudo ha presentado una tendencia decreciente durante los últimos diez años [2].

En este sentido, el biodiesel se presenta como una alternativa para disminuir el impacto ambiental de los combustibles tradicionales. Sin embargo, el proceso convencional de transesterificación de aceites para la obtención de biodiesel da como resultado la formación de glicerina como producto secundario en un $10 \%$ con respecto a la materia prima suministrada, según Demirbas [3]. Actualmente se conocen múltiples aplicaciones posibles para la glicerina y sus derivados, que se comercializan según sus grados de calidad; pero lo cierto es que no existen aún propuestas de uso directo definidas para la glicerina obtenida como subproducto del biodiesel, debido a que en general esta no cumple el estándar mínimo de calidad y su costo de refinación a grado USP (Grado Farmacéutico y Alimenticio), conduce a un producto poco viable, según Yang y colaboradores [4].

Por esta razón, se ha motivado la investigación de otras estrategias de recuperación de recursos, siendo una opción la bioconversión de glicerina a compuestos de alto valor a través de fermentación microbiana anaeróbica, ya que por su mayor grado de reducción de azúcares ofrece la oportunidad de obtener productos químicos como el succinato, etanol, xilitol, propionato, hidrógeno, etcétera, lo que representa una vía prometedora para lograr la viabilidad económica en la industria de los biocombustibles.

\subsection{Glicerina, subproducto de la producción de biodiesel}

La glicerina cruda, con una concentración máxima del $60 \%$ de glicerina, es de poco valor, ya que contiene gran cantidad de jabones, catalizador alcalino y metanol, y es medioambientalmente peligrosa, debido a este último compuesto; por tanto, para poder aprovecharla, debe llevarse al menos a un grado técnico $(85 \% \mathrm{p} / \mathrm{p}$ de pureza), según Hasheminejad y colaboradores [5].

Una alternativa común es someterla a un proceso de acidulación con ácido fosfórico, para separar las tres fases presentes: la glicerina (con el metanol aún disuelto); los ácidos grasos libres (provenientes del aceite); y una fase sólida consistente de sales formadas por la reacción entre el catalizador alcalino y el ácido agregado [6].

La glicerina resultante debe ser separada del metanol hasta alcanzar una pureza de aproximadamente $85 \%(\mathrm{p} / \mathrm{p})$. En esta condición ya está lista para su uso directo o como insumo en otros procesos industriales [7].

\subsection{Usos alternativos de la glicerina}

Encontrar nuevos usos de la glicerina cruda de la industria del biodiesel sigue siendo motivo de preocupación entre los productores actuales y futuros. Sin embargo, la selección e implementación de cualquiera de las alternativas de aprovechamiento, requiere de un estudio global que permita concluir sobre la viabilidad del proceso [4]. Asimismo, los principales productos de una biorrefinería son energéticos en forma de calor, electricidad o biocombustibles, y otros biomateriales de alto valor agregado en el mercado, lo que permite la rentabilidad del proceso. Como ejemplos de biomateriales se pueden citar proteínas, enzimas, ácidos orgánicos, colorantes, antioxidantes, prebióticos, agentes inmunológicos y saborizantes. Esta amplia gama de biomateriales se debe a la diversidad en los tipos de biomasa disponibles y a las tecnologías que pueden ser utilizadas en una biorrefinería para la producción de etanol, a partir de glicerina obtenida como subproducto de la producción de biodiesel.

Igualmente, la glicerina se ha convertido en una materia prima ideal para producir compuestos reducidos a través de la fermentación anaeróbica. Yazdani y González [8] identificaron las condiciones medioambientales que permiten la fermentación de glicerina, junto con las vías y los mecanismos que median este 
proceso para la conversión eficiente de glicerina cruda en etanol, a un costo menor que los procesos tradicionales que utilizan la caña de azúcar.

\subsection{Fermentación}

La fermentación es un proceso típico que se lleva a cabo en un fermentador o, en general, biorreactor, mediante el cual determinados sustratos que componen el medio de cultivo son transformados por acción microbiana en metabolitos y biomasa. Existen cuatro etapas bien diferenciadas: 1. propagación de cultivos, lo que se realiza en el laboratorio y que comienza generalmente en un tubo de ensayo que contiene un repique reciente del microorganismo o un tubo liofilizado o congelado, donde se conserva la cepa de interés o de una colonia del microorganismo previamente seleccionada; 2. fermentación, con el material obtenido anteriormente, se siembra el tanque de inóculo que puede tener un volumen de 50, 500 o $1000 \mathrm{~L}$ según la escala industrial posterior; del tanque de inóculo se pasa posteriormente al fermentador industrial cuyo volumen puede variar de acuerdo con el producto que se va a obtener; 3. operaciones y proceso de separación y purificación de los productos. Estas etapas comprenden la separación de insolubles por filtración, centrifugación o decantación, posteriormente, separaciones primarias por extracción, absorción, adsorción, ultrafiltración y, por último, purificación por extracción líquido-líquido, o extracción a dos fases acuosas, o cromatografía de afinidad hasta el aislamiento del producto; 4 . tratamiento de efluentes [9].

Las levaduras son los microorganismos más utilizados en la producción de etanol por la vía fermentativa, debido a que producen un mejor proceso de separación después de la fermentación, además producen un contenido de toxinas muy inferior a otros microorganismos [10].

Tal es el caso de la levadura Saccharomyces cerevisiae, que bajo condiciones anaerobias reduce el piruvato a etanol con emisiones de $\mathrm{CO}_{2}$, obteniéndose un rendimiento estequiométrico teórico de $0,511 \mathrm{~g}$ de etanol y 0,489 g de CO, por $1 \mathrm{~g}$ de glucosa metabolizada. Sin embargo, se ha observado que a nivel experimental e industrial sólo se alcanza entre el $87 \%$ y el $95 \%$ del rendimiento teórico para el etanol, ya que esta levadura también utiliza la glucosa en la producción de otros subproductos. No obstante, se prefiere su uso por requerir de menores costos de manejo, ya que la presencia de sacarosa en algunos sustratos no afecta el ren- dimiento de etanol; asimismo, los subproductos de la fermentación y la concentración a la cual estos se producen no crean efectos colaterales [11].

Dentro de los requerimientos nutricionales, de las fuentes de carbono y energía que puede emplear la Saccharomyces cerevisiae figuran algunos monosacáridos como la glucosa, fructosa, manosa y galactosa, entre otros. También son capaces de utilizar disacáridos como la maltosa y la sacarosa y trisacáridos como la rafinosa. Además de utilizar otras fuentes de carbono distintas a carbohidratos y aminoácidos, entre las más destacadas se encuentra la capacidad de utilizar la glicerina [12].

\subsection{Etanol}

Estudios realizados permitieron obtener etanol al 99,5\% de pureza, a partir del diseño del proceso de fermentación anaeróbica de glicerina cruda a etanol [13]. Esto es concordante con los estudios de Ito y colaboradores [14] y Dharmadi y colaboradores [15], quienes adicionalmente describen el proceso en etapas, las cuales principalmente consisten en la preparación previa de glicerina crudo de fermentación, la fermentación de la glicerina en etanol y el ácido succínico, y la separación secundaria de los productos mediante destilación, tamices moleculares u otras unidades de separación.

En 2012, Kasavi y colaboradores [16] evaluaron cinco cepas de Saccharomyces cerevisiae industriales por su sostenibilidad para uso en los procesos de producción de etanol. El crecimiento y el rendimiento de producción de etanol se valoró en diversos residuos agroindustriales -incluyendo pasta de azúcar de remolacha, almidón y melaza de remolacha azucareray residuos biológicos - como la zanahoria, tomate y patatas-. Unos análisis de componentes principales de los parámetros de fermentación relacionados con el crecimiento y la producción de las cinco cepas cultivadas en glucosa demostraron la superioridad de una de las cepas (Y9) respecto a su rápido crecimiento y los rendimientos más altos de etanol.

De igual forma, una cepa de Saccharomyces cerevisiae fue metabólicamente diseñada para mejorar la producción de etanol a partir de glicerol. Las altas tasas de utilización de glicerol se lograron por la sobreexpresión simultánea de glicerol deshidrogenasa (gcy) y dihidroxiacetona quinasa (Dak). La producción de etanol en general en la cepa YPH 499 (pGcyaDak, pGupCas) fue 3,4 veces más que en la cepa salvaje, con alrededor de $2,4 \mathrm{~g} / \mathrm{L}$ etanol producido [10]. De la misma mane- 
ra, en estudios posteriores, Yu y colaboradores [17] demostraron la posibilidad de proporcionar protección contra la presión osmótica y al mismo tiempo aumentar etanol y la reducción de la producción de glicerol en cepas de $S$. cerevisiae, utilizando glicerina como fuente de carbono.

Asimismo, Ochoa-Estopier y colaboradores [18] demuestran que el crecimiento sucesivo de la $S$. cerevisiae CBS 8066, CEN.PK 113-7D en glicerina como única fuente de carbono mejoró considerablemente la producción de etanol desde 0,01 hasta $0,2 \mathrm{~h}^{-1}$.

\section{Metodología}

Se llevará a cabo un estudio de tipo experimental para determinar cuál es el porcentaje de etanol obtenido a partir de glicerina purificada, necesario para el diseño de un prototipo a escala industrial mediante procesos fermentativos, utilizando Saccharomyces cerevisiae. Se analizarán variables de importancia en un proceso fermentativo discontinuo o batch, monitoreado durante 36 horas. De esta forma, el proyecto involucrará cinco fases para seguir así:

1. Activación y establecimiento de las cepas de Saccharomyces cerevisiae: sembrando el microorganismo en Agar Sabouraud e incubándolo a $37^{\circ} \mathrm{C}$ por 48 horas, para el establecimiento de la cepa madre; seguido por un recuento en cámara de Neubauer para evaluar la pureza de la misma.

2. Determinación del comportamiento cinético de Saccharomyces cerevisiae: se establecerán cuatro tratamientos experimentales (T1, T2, T3) y un tratamiento control (T0) que se monitorearán con intervalos de tres horas durante 36 horas, evaluándose variables como concentración de biomasa por recuento directo en cámara de Neubauer, $\mathrm{pH}$ que permitirán determinar parámetros cinéticos de importancia.

3. Establecimiento de las condiciones de proceso para la optimización del medio de cultivo y estandarización de la fermentación: aquí se determinará la temperatura óptima para incrementar la producción de este metabolito (etanol) en el medio de cultivo a evaluar teniendo en dos condiciones de temperatura $10-15^{\circ} \mathrm{C}$ y de $30-35^{\circ} \mathrm{C}$.
4. Rendimiento de producción de etanol, a partir de los microorganismos, a partir de la glicerina: que involucrará el análisis de los resultados obtenidos anteriormente mediante un análisis ANOva multifactorial, determinando si existen diferencias entre los tratamientos según el porcentaje de etanol producido.

5. Modelación del proceso a escala industrial: donde a partir de los resultados obtenidos anteriormente a escala de laboratorio, se podrá simular a una escala mayor el proceso de producción de etanol y verificar la rentabilidad del proceso. Como resultado de esta investigación se espera establecer el dimensionamiento de los equipos para la producción de etanol, por medios fermentativos a partir de glicerina cruda y purificada.

\section{Discusión y conclusiones}

Con la ejecución del proyecto de investigación se pretende evaluar el comportamiento de las variables técnicas más importantes para la producción de etanol, a partir de glicerina, obtenida como subproducto de la producción de biodiesel, buscando la activación de las cepas de Saccharomyces cerevisiae, la determinación del comportamiento cinético, el establecimiento de condiciones de proceso para la optimización del medio de cultivo y estandarización de la fermentación, así como la simulación de los procesos que permiten realizar el diseño del proceso, teniendo en cuenta las propiedades fisicoquímicas, los balances de materia y energía y las unidades de operación requeridas para el modelamiento del proceso con un alto grado de confiabilidad, y obteniendo mayor entendimiento de cada aspecto del proceso productivo.

Lo anterior es en buena medida un reflejo de la necesidad científica que existe de encontrar y desarrollar tecnologías que permitan darle uso a la gran oferta disponible de glicerina, provocada por el auge de biodiesel a nivel mundial, aunado a los precios bajos de este subproducto.

Al utilizar la glicerina como materia prima para la producción de etanol, se obtiene un valor agregado a la producción de biodiesel de aceite de palma, en función de alternativas amigables con el medioambiente, proporcionándole una alternativa de aprovechamiento a este subproducto y un biocombustible de segunda generación. 


\section{Referencias}

[1] Gobierno Federal-Secretaría de Energía, Prospectiva del mercado de petróleo crudo 2010-2025. México, 2011. [En Línea]. Disponible en: http://www.sener.gob.mx

[2] Asociación Colombiana de Petróleo, "Informe estadístico petrolero 2011”, Bogotá, 2012. [En línea]. Disponible en: http://www.acp.com.co

[3] A. Demirbas, "Biodiesel A Realistic Fuel Alternative for Diesel Engines”, Springer London, 2008.

[4] F. Yang, M. Hanna, y R. Sun, "Value-added uses for crude glycerol-a byproduct of biodiesel production". Biotechnology for Biofuels, Vol. 5, pp. 1-10, No. 13, 2012.

[5] M. Hasheminejad, M. Tabatabaei, Y. Mansourpanah, M. Khatami y A. Javani, "Upstream and downstream strategies to economize biodiesel production". Bioresource Technology, Vol. 102, No. 2, pp. 461-468, 2011.

[6] A. Singhabhandhu y T. Tezuka, "A perspective on incorporation of glycerin purification process in biodiesel plants using waste cooking oil as feedstock". Energy, Vol. 35, pp. 2493-2504, 2010.

[7] M. Hajek y F. Skopal, “Treatment of glycerol phase formed by biodiesel production". Bioresource Technology, Vol. 101, No. 9, pp. 3242-3245, 2010.

[8] S. Yazdani y R. Gonzalez, "Anaerobic fermentation of glycerol: a path to economic viability for the biofuels industry". Current opinion in biotechnology. Vol. 7, No. 18, pp. 213-219, 2007.

[9] W. Crueger y A. Crueger, Biotecnología: Manual de Microbiología Industrial, Zaragoza: Editorial Acribia, 1993, pp. 143-146.

[10] K. Yu, S. Kim y S. Han, "Engineering of glycerol utilization pathway for ethanol production by Saccharomyces cerevisiae". Bioresource Technology, Vol. 101, pp. 4157-4161, 2010.
[11] C. Cardona y C. Orrego, Avances investigativos en la producción de biocombustibles, Manizales: Universidad Nacional de Colombia, 2009.

[12] N. Akhtar, A. Pahlman, K. Larsson, A. Corbett y L. Adler, "SGD1 encodes an essential nuclear protein of Saccharomyces cerevisiae that affects expression of the GPD1 gene for glycerol 3-phosphate dehydrogenase", FEBS Lett. Vol. 483, No. 2-3, pp. 87-92, 2000.

[13] R. González, A. Murarka, Y. Dharmadi, y S. Yazdani, "A new model for the anaerobic fermentation of glycerol in enteric bacteria: Trunk and auxiliary pathways in Escherichia coli”. Metabolic Engineering, Vol. 10, No. 5, pp. 234-245, 2008.

[14] T. Ito, Y. Nakashimada, K. Senba, T. Matsui y N. Nishio, "Hydrogen and Ethanol Production from Glycerol - Containing Wastes Discharges after Biodiesel Manufacturing Process". Journal of Bioscience and Bioengineering, Vol. 100, No. 3, pp. 260-265, 2005.

[15] Y. Dharmadi, A. Murarka, R. Gonzalez y S. Yazdani, "A new model for the anaerobic fermentation of glycerol in enteric bacteria: Trunk and auxiliary pathways". Escherichia coli. Metabolic Engineering, Vol. 10, No. 5, pp. 234-245, 2008.

[16] C. Kasavi, I. Finore, L. Lama, B. Nicolaus, G. Oliver, E. Toksoy y B. Kirdar, "Evaluation of industrial Saccharomyces cerevisiae strains for ethanol production from biomass". Biomass and Bioenergy, Vol. 45, pp. 230-238, 2012.

[17] K. Yu, S. Kim y S. Han, "Reduction of glycerol production to improve ethanol yield in an engineered Saccharomyces cerevisiae using glycerol as a substrate". Journal of Biotechnology, Vol. 150, pp. 209-214, 2010.

[18] A. Ochoa-Estopier, N. Lesage, N. Gorret y S. Guillouet, "Kinetic analysis of a Saccharomyces cerevisiae strain adapted for improved growth on glycerol: Implications for the development of yeast bioprocesses on glycerol". Bioresource Technology, Vol. 102, pp. 1521-1527, 2011. 